\title{
A Thermal-Bubble-Actuated Micronozzle-Diffuser Pump
}

\author{
Jr-Hung Tsai and Liwei Lin, Member, IEEE
}

\begin{abstract}
A thermal-bubble-actuated micropump by the principles of liquid/vapor phase transition and nozzle-diffuser flow regulation is successfully demonstrated. The micropump consists of a resistive heater, a pair of nozzle-diffuser flow controller and a $1 \mathrm{~mm}$ in diameter, $50 \mu \mathrm{m}$ in depth pumping chamber. The actuation mechanism comes from periodically nucleating and collapsing thermal bubbles. A net flow is generated from the nozzle to the diffuser by the nozzle-diffuser flow controller. Two heater designs, single-bubble and dual-bubble actuation mode, have been investigated. In the single-bubble pumping mode, a maximum flow rate of $5 \mu \mathrm{l} / \mathrm{min}$ is measured when the driving pulse is $250 \mathrm{~Hz}$ at $10 \%$ duty cycle under an average power consumption of $1 \mathrm{~W}$. A similar flow rate of $4.5 \mu \mathrm{l} / \mathrm{min}$ is achieved in the dual-bubble pumping mode, at the driving pulse of $5 \%$ duty cycle at $400 \mathrm{~Hz}$ with lower average power consumption, $0.5 \mathrm{~W}$. The static pumping pressure is measured at a maximum value of 377 Pascal when the net volume flow rate is zero. As an application example in a microfluidic device, this valve-less micropump is used in a microfluidic system to enhance the fluid mixing by agitating the flows.

[742]
\end{abstract}

Index Terms-Bubble, microfluidics, mixing, phase change, pump.

\section{INTRODUCTION}

$\mathbf{M}$ ICROPUMPS have wide applications in MEMS devices, such as handling small and precise volume of fluids for chemical, biological or medical systems. Many design concepts and prototype micropumps have been demonstrated over the past decade and they can be classified into following types by means of actuation mechanisms: mechanical displacement [1]-[3], bubble [4], electrohydrodynamic [5], and electrocapillary [6]. Among these actuation methods, bubble actuation can be easily generated by resistive heaters and can be applied to nonspecific liquids, thus is an attractive actuation source in the micro scale. Existing device examples include bubble-jet printers [7] and bubble powered micro mechanical actuators [8].

On the other hand, micro valves are indispensable components in microfluidic systems for flow rate and flow direction control such that they are usually integrated with micropumps [3], [9]-[11]. Unfortunately, micro valves generally increase the complexity of the fabrication process and the possibility of par-

Manuscript received August 21, 2001; revised February 8, 2002. This work was supported in part by an NSF CAREER AWARD (ECS-0096098) and a DARPA/MTO/BioFlips grant. Subject Editor G. Stemme.

J.-H. Tsai was with Mechanical Engineering, University of Michigan, Ann Arbor, MI USA. He is now with the Mechanical Engineering Department, University of California, Berkeley, CA 94720-1740 USA (e-mail: jhtsai@argon.eecs.berkeley.edu).

L. Lin is with the Mechanical Engineering Department, University of California, Berkeley, CA 94720-1740 USA.

Digital Object Identifier 10.1109/JMEMS.2002.802909

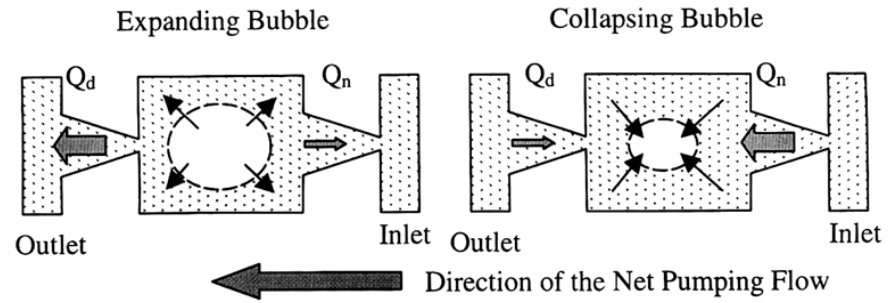

Fig. 1. Pumping principle illustrated from the top view of the pumping chamber.

ticle clogging and contamination in the microfluidic pathways. These shortcomings make valve-less pumping attractive as an alternative way for the micropump design [2], [12], [13]. A valve-less micropump by means of nozzle-diffuser design and piezoelectrical actuation was developed previously [2]. The efficiency of this micropump depends largely on the deflection magnitude and driving frequency of the mechanical membrane. It becomes significantly difficult to further miniaturize these diaphragm-based pumps because the large force is required to deflect the diaphragms for pumping.

This paper applies the principle of thermal bubble nucleation for pumping and the nozzle-diffuser flow controller design to direct the induced oscillatory flow. As such, the pumping efficiency can be improved because: 1) thermal bubbles can be easily generated even when the pump is further miniaturized and 2) thermal bubble can sweep across most of the pumping chamber for optimal efficiency. Furthermore, the concern of complicated fabrication process for micro valves is alleviated because there are no moving mechanical parts in the micropump.

\section{Micropump Design, FABRICATION, AND EXPERIMENTAL SETUP}

\section{A. Design}

Fig. 1 illustrates the fundamental design concept of the micropump. Micro bubble is generated by thermal bubble nucleation in the micro chamber to create a pumping pressure source. As the pumping bubble expands, the volume flow rate at the diffuser, $Q_{d}$, is larger than the one at the nozzle, $Q_{n}$. When the pumping bubble collapses, $Q_{d}$ is smaller than $Q_{n}$. Therefore, there is a net flow generated from the nozzle to diffuser each cycle as shown. The boundary of the bubble works as a membrane that sweeps across the chamber to minimize the pumping dead volume. Furthermore, because of the nozzle-diffuser design, no conventional mechanical valve is used in the operation such that the fabrication process is simplified. 


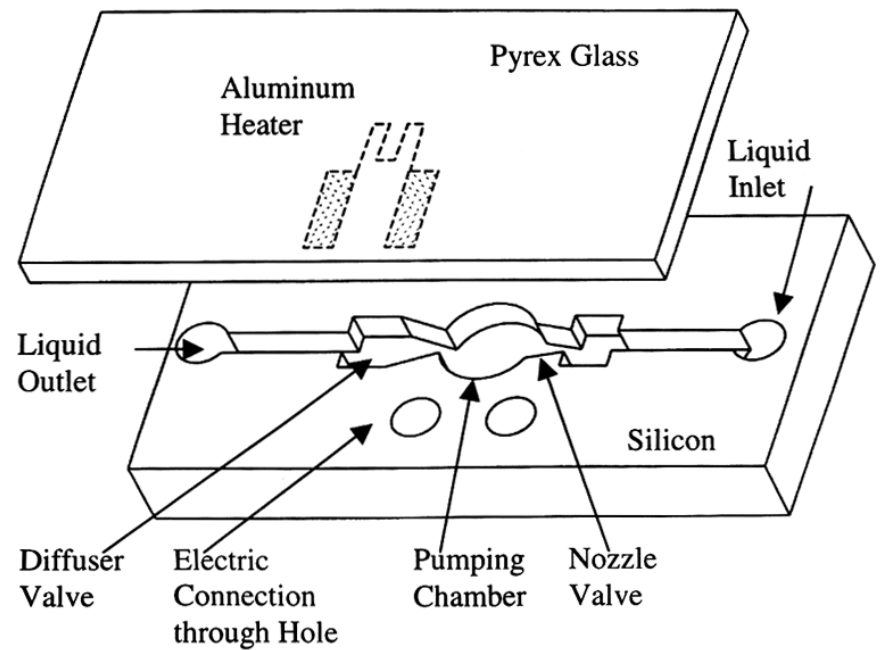

Fig. 2. Schematic drawing of the nozzle-diffuser based bubble pump.

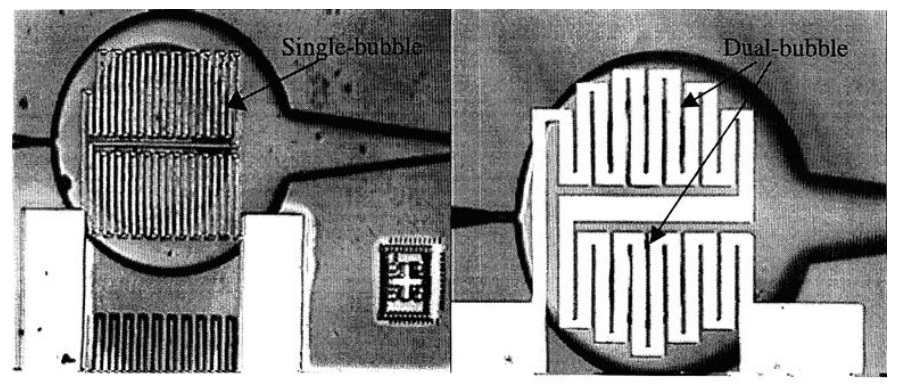

(a)

(b)

Fig. 3. Two types of heater patterns, (a) single-bubble mode and (b) dualbubble mode.

Based on the operation principle, the micropump is fabricated with a micromachining process as schematically displayed in Fig. 2. The pumping chamber, nozzle-diffuser flow controller and channels, are fabricated on a silicon substrate by the same depth of $50 \mu \mathrm{m}$ using DRIE (Deep Reactive Ion Etching). Through holes for liquid inlet and outlet ports and electrical interconnection are also fabricated on the silicon substrate by using another DRIE process. A Pyrex glass wafer is used to seal the microsystem and aluminum is evaporated and patterned on it as shown in Fig. 2 as the resistive heater. A meander-shape heater is selected rather than a solid rectangular plate-shape heater (commonly used in bubble jet printers) such that bubble nucleation in the pumping chamber can be observed from the glass side. In the prototype trial, the selection of the dimensions is based on the previous report of a piezoelectrically driven, nozzle-diffuser micropump [14]. The circular pumping chamber is chosen as $1 \mathrm{~mm}$ in diameter and is connected with a pair of nozzle-diffuser flow controller of $30 \mu \mathrm{m}$ in the narrow neck, $274 \mu \mathrm{m}$ in the open mouth and the diverging angle is $14^{\circ}$. The fluid channels that connect the micropump to the fluid inlet/outlet ports are $200 \mu \mathrm{m}$ in width. Two polyimide tubes of $310 \mu \mathrm{m}$ in outer diameter and $275 \mu \mathrm{m}$ in inner diameter are used to connect the micropump to the macro world via the etched through liquid inlet/outlet ports on the silicon substrate. Fig. 3(a) and (b) display two optical photos of fabricated micropumps of single-bubble pumping mode and dual-bubble

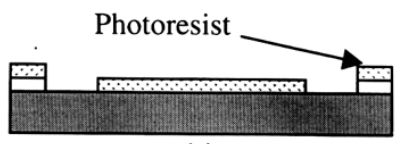

(a)

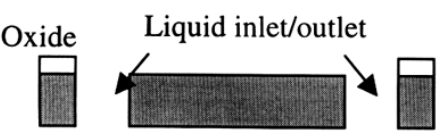

(b)

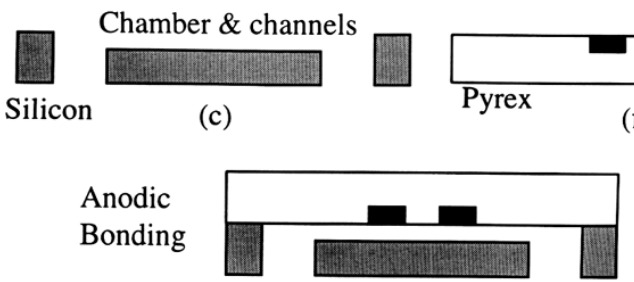

(g)

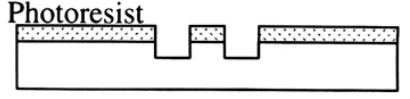

(d)

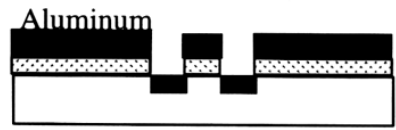

(e)

(f)
Fig. 4. Fabrication process.

pumping mode, respectively. In the single-bubble pumping mode, the heater is designed to generate one bubble group in the center of the pumping chamber. In the dual-bubble pumping mode, the heater is designed to generate two bubble groups simultaneously that can be barely observed in Fig. 3(b).

\section{B. Fabrication}

The fabrication process is illustrated in Fig. 4. On the silicon substrate, a two-step DRIE process is used. The silicon wafer is deposited with a $1-\mu \mathrm{m}$-thick thermal oxide that is later patterned and etched as the oxide mask to define the pumping chamber, nozzle-diffuser pair and microchannels. A 9- $\mu$ m-thick photoresist is spun on, patterned as the photoresist mask to define through holes for the liquid inlet/outlet ports and electrical interconnections as shown. After these steps, Fig. 4(a) applies. The first DRIE process is conducted afterwards to etch through the whole silicon wafer and the photoresist mask is removed as shown in Fig. 4(b). The second DRIE process is carried out with the oxide mask and about $50 \mu \mathrm{m}$ deep of silicon is etched away. Afterwards, the silicon wafer is dipped into hydrofluoric acid (HF) to remove silicon dioxide and the silicon wafer is ready for assembly as shown in Fig. 4(c). On the Pyrex glass substrate, recesses of about $1 \mu \mathrm{m}$ in depth for embedding aluminum heaters are formed to prevent liquid leakage around the electrodes and to ensure good anodic bonding result. Photoresist is used to define the patterns and buffered HF is used as the etchant to form the recesses as shown in Fig. 4(d). Aluminum is then evaporated and patterned as resistive heaters by the lift-off process as shown in Fig. 4(e) and (f), respectively. Finally, The silicon and Pyrex wafers of $500 \mu \mathrm{m}$ in thickness are aligned and anodically bonded at $380^{\circ} \mathrm{C}$ in Fig. $4(\mathrm{~g})$.

\section{Experimental Setup}

In the proof-of-concept experiments, ceramic particles with size of 3-15 $\mu \mathrm{m}$ in diameter are mixed with isopropyl alcohol (IPA) as the pumping liquid. IPA is chosen as the test liquid for its easy access and low boiling temperature. By tracing the 


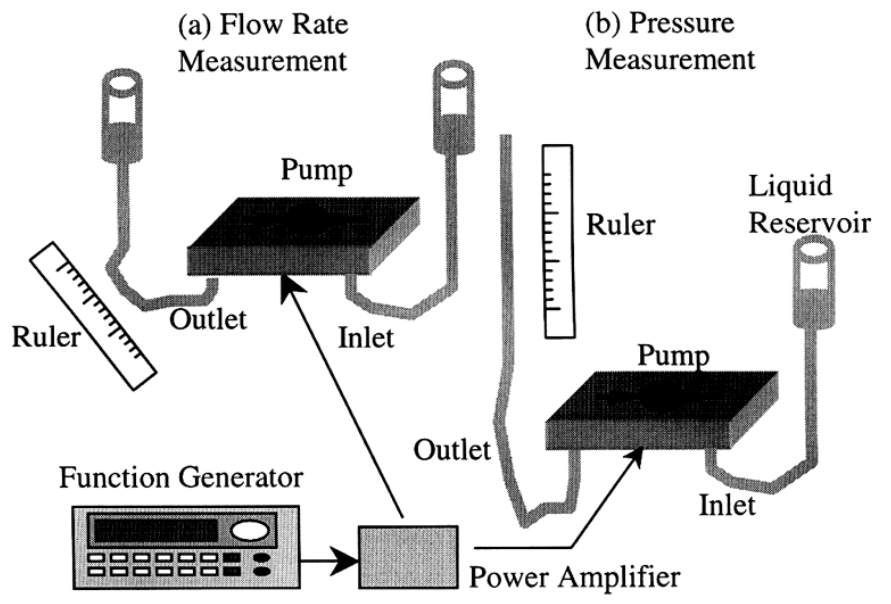

Fig. 5. Schematic drawing of the experimental setups. (a) Flow rate measure under zero back pressure. (b) Pumping pressure under zero flow rate.

movement of the particles, the net flow speed is estimated. It is observed that the liquid is oscillating in the same frequency as the actuation pulse even in the interconnecting polyimide tubes. When the micropump is operating at frequencies higher than $10 \mathrm{~Hz}$, it becomes difficult to trace particle movement with a regular image recorder of 25 frame/s.

The experimental setups for both the volume flow rate and pumping pressure measurements are illustrated in Fig. 5. In both experiments, the far ends of the polyimide tubes from the micropump are connected to syringes filled with IPA as liquid reservoirs. In the volume flow rate measurement as shown in Fig. 5(a), the two syringes are set at the same height for equilibrium pressure. The pumping flow rate is measured from the averaged speed of an air slug that is placed into the polyimide tube in advance. Volume flow rate data are calculated from the average speeds of the air bubble travelling a distance of $1 \mathrm{~cm}$ in each case. The syringes have inner diameter of $14 \mathrm{~mm}$ which is much larger than the diameter of the polyimide tube. Therefore, the liquid level change in the syringe during the pumping process is negligible and the measured volume flow rate is assumed to be under zero back pressure.

In the pumping pressure measurement shown in Fig. 5(b), the back pressure that stops the net flow is recorded. The setup is similar to the one for measuring volume flow rate except that there is no syringe at the outlet port of the pump. Liquid level in equilibrium is reached before the pump is turned on. The pumping pressure is then calculated from the liquid level change in the outlet polyimide tube when the maximum height is reached.

\section{RESULTS AND DISCUSSIONS}

Fig. 6 shows two sequential pictures taken 8 seconds apart at about $1 \mathrm{~mm}$ upstream from the nozzle during the particle tracing experiment. Particles were observed oscillating coherently to the bubble expanding/collapsing cycle at the pulse excitation frequency of $3 \mathrm{~Hz}$, duty cycle of $5 \%$ and average power consumption of 0.05 Watt. At the neck of the nozzle, particle traces can hardly be observed during the bubble expanding process due to the high flow speed. As time goes by, particles can be observed to have different net displacements. It indicates the

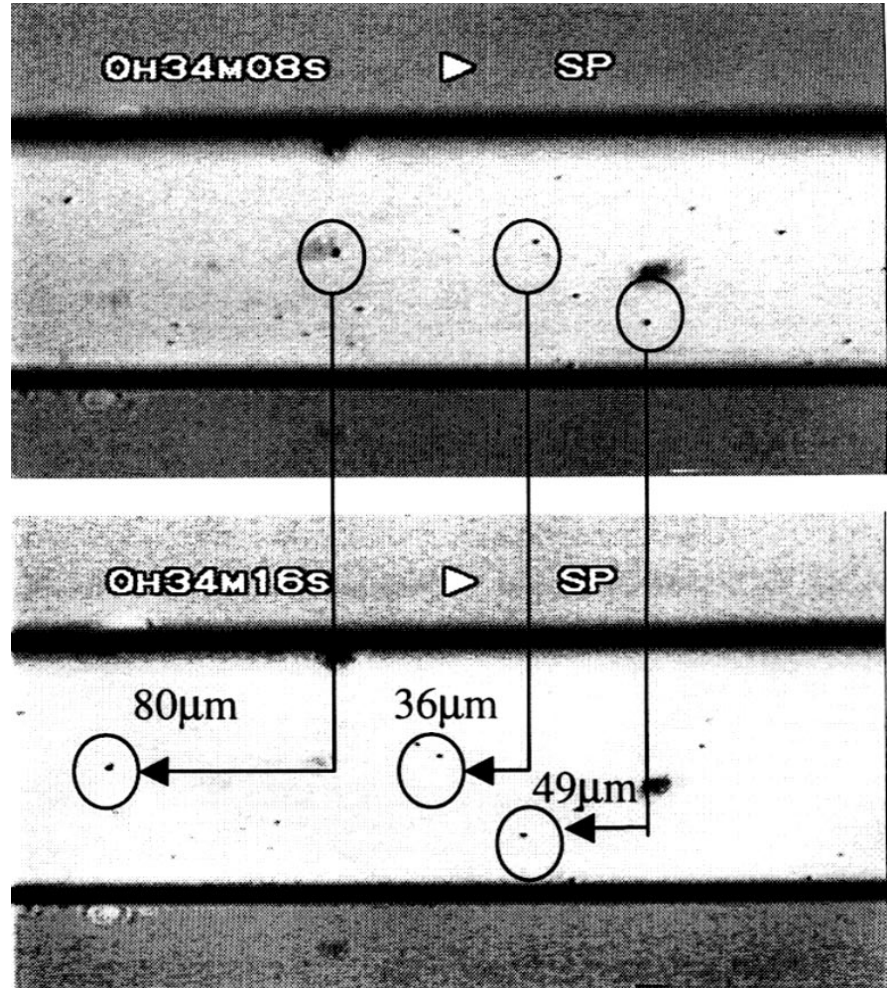

Fig. 6. Two sequential pictures showing particle movement in 8 seconds under an excitation frequency of $3 \mathrm{~Hz}$.

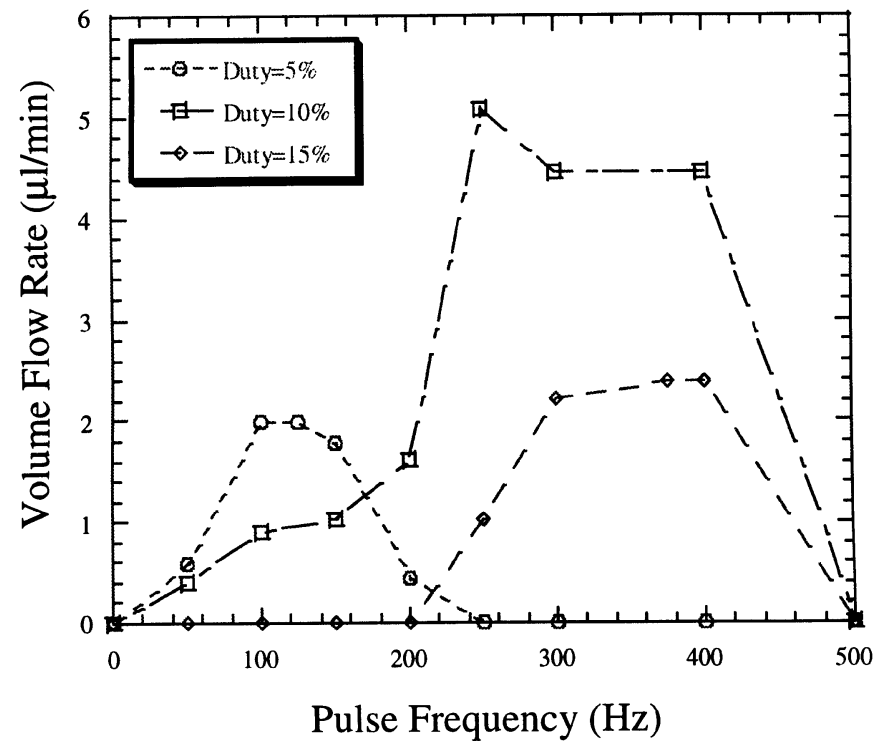

Fig. 7. Volume flow rate of single-bubble pumping mode under pulse voltage of $20 \mathrm{~V}$.

nonuniform velocity profile across the microchannel. An averaged velocity of $6.9 \mu \mathrm{m} / \mathrm{s}$ that corresponds to a volume flow rate of $3.7 \mathrm{nl} / \mathrm{min}$ is estimated by tracing the circled particles in a period of $8 \mathrm{~s}$.

When the driving frequency is increased, Fig. 7 shows the measured flow rates in the single-bubble pumping mode actuated by three different square pulse duties, $5 \%, 10 \%$, and $15 \%$ over a frequency spectrum up to $500 \mathrm{~Hz}$. The applied pulse voltage is $20 \mathrm{~V}$ and current is $0.5 \mathrm{~A}$. The maximum pumping 


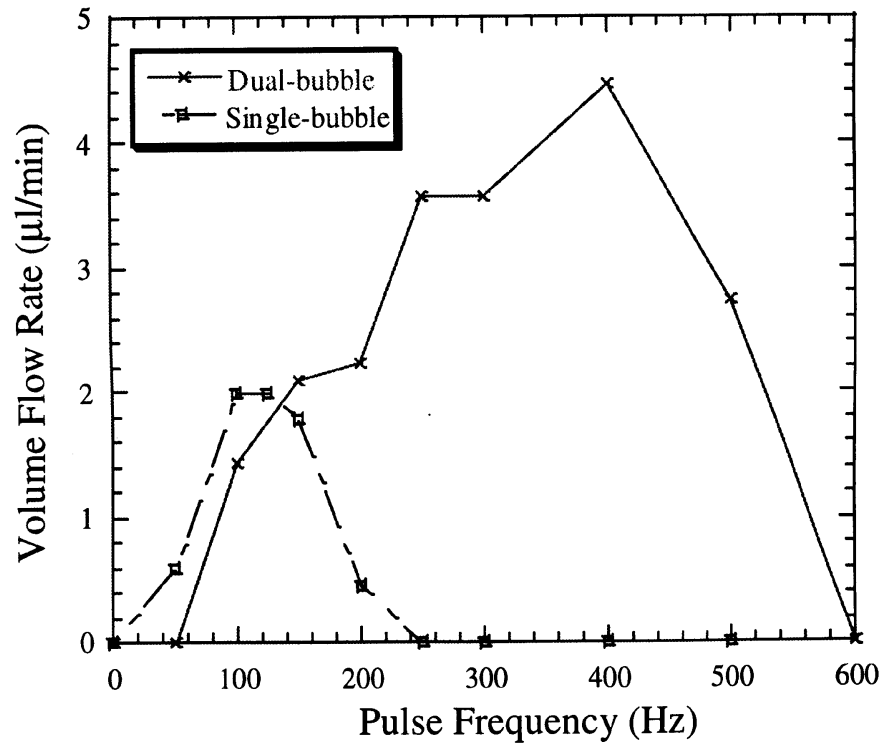

Fig. 8. Volume flow rate by single- and dual-bubble pumping modes at $5 \%$ duty cycle and $0.5 \mathrm{~W}$ average input power.

rate is found as $5 \mu \mathrm{l} / \mathrm{min}$ when the pulse duty is $10 \%$ and the pulse frequency is $250 \mathrm{~Hz}$. According the model developed by Olsson et al. [15], the volume flow rate $Q$ of nozzle-diffuser pumps can be modeled as

$$
Q=2 V f\left[\frac{\left(\frac{\xi_{n}}{\xi_{d}}\right)^{1 / 2}-1}{\left(\frac{\xi_{n}}{\xi_{d}}\right)^{1 / 2}+1}\right]
$$

where $V$ is the variation of liquid volume in the chamber per pumping cycle, $f$ is the pulse frequency, and $\xi_{n}$ and $\xi_{d}$ are the coefficients of pressure loss of the nozzle and diffuser, respectively. The thermal bubble based nozzle-diffuser pumping system represents a rather complicated thermodynamic problem including heat transfer in the microscale, bubble nucleation and collapsing, fluidic reaction of the nozzle-diffuser design. A general discussion based on the experimental results of Fig. 7 is made with the assistance of (1). First, it is assumed that the ratio of coefficients of pressure loss of the nozzle and diffuser is constant during the operation. When the driving frequency is increased, the flow rate increases proportionally as indicated by (1) and the experimental results follow the trend. However, this prediction is based on the assumption that the bubble pumping volume per cycle remains constant. Two factors contradict this assumption. First, at very high driving frequency, the bubble forms and persists like the result of a dc input. Without the collapsing of bubbles at each cycle, the pumping volume per cycle, $V$, reduced dramatically and to zero when the chamber is completely filled with a residual bubble. Second, when the duty cycle is high and the pumping frequency is low, such as the case of $15 \%$ duty cycle at frequencies lower than $200 \mathrm{~Hz}$ in Fig. 7. It is observed that a large residual bubble can not be collapsed during the operation, thus the pumping flow rate is reduced and undetectable. This phenomenon is believed to be the result of heat accumulation under high duty cycle and low operation frequency that prevents the bubble from collapsing.

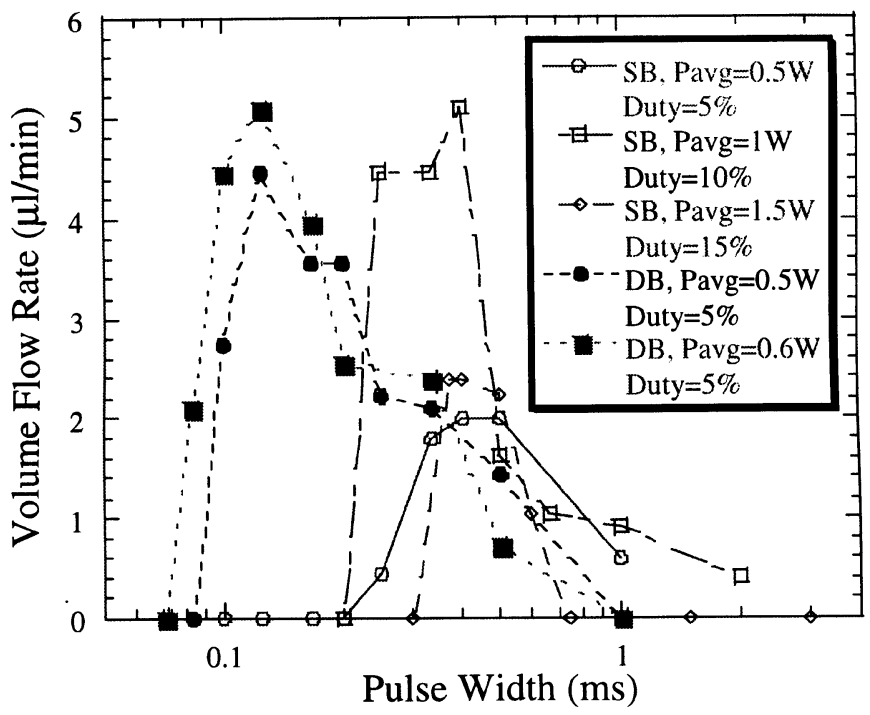

Fig. 9. Volume flow rate versus pulse width by single-bubble (SB) and dual-bubble (DB) pumping mode under various average input power.

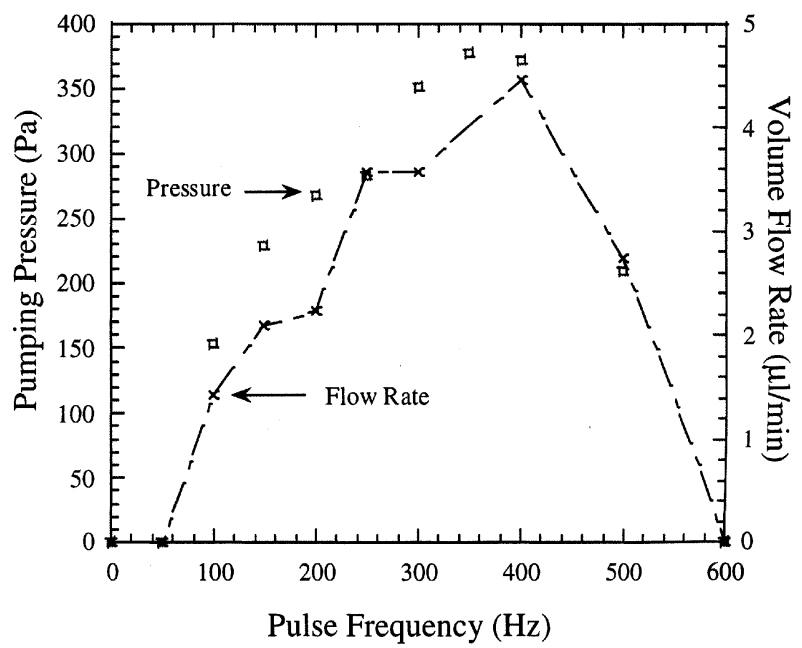

Fig. 10. Pumping pressure at zero flow rate and volume flow rate at zero back pressure under dual-bubble pumping mode, $5 \%$ pulse duty and $0.5 \mathrm{~W}$ averaged input power.

In Fig. 8, the flow rate in the single-bubble pumping mode is compared with the flow rate in the dual-bubble pumping mode. In both cases, bubbles are roughly distributed in one and two groups in the single and dual-bubble pumping modes, respectively, as illustrated in Fig. 3. The peak flow rate generated by the dual-bubble pumping mode is higher than the single-bubble one under the same average driving power. The possible reason is that the design in the dual-bubble pumping mode alleviates the overheating and residual bubble problem such that the maximum flow rate is increased and achieved at a higher pumping frequency of $400 \mathrm{~Hz}$.

The pumping flow rate relies on the many factors, such as the input power, duty cycle and driving frequency. Fig. 9 shows the measured flow rates with respect to pulse widths that is the product of the pulse duty multiplied with the reciprocal of pulse frequency. It is observed that when the pulse width is around 0.4 $\mathrm{ms}$ for the single-bubble pumping mode and 0.13 millisecond for the dual-bubble pumping mode, the pumping flow rates 


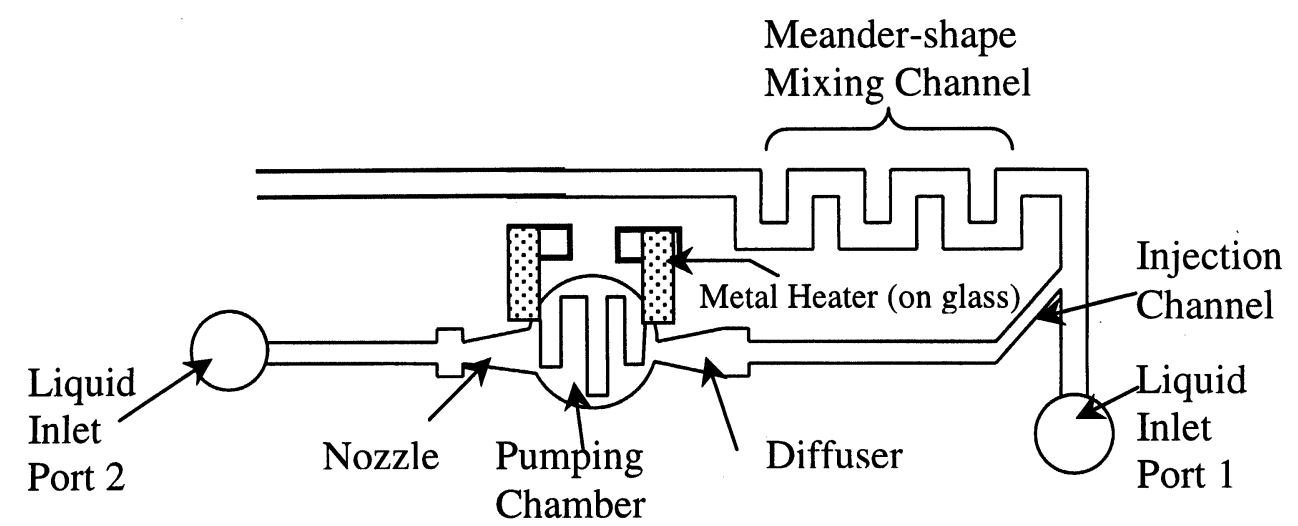

Fig. 11. Schematic drawing of the exemplary integrated system of a micropump and a mixing channel.

reaches maximum values, respectively. The observation implies that pulse width plays the dominant role in achieving maximum pumping flow rate.

On the other hand, it is difficult to evaluate the effect of input power without establishing a three-dimensional (3-D) heat transfer model that involves phase change, thermodynamics and fluid reaction in the micro scale. The observation from Fig. 9 is that the increment in input power helps increasing the pumping flow rate before the input power is so high that generates residual bubbles (such as the case of single-bubble pumping mode with average input power of $1.5 \mathrm{~W}$ ). It is also observed that the dual-bubble pumping mode has higher maximum pumping rates when compared with the single-bubble pumping mode under a similar average input power. Furthermore, only a smaller pulse width is required to generate the maximum pumping flow rate in the case of the dual-bubble pumping mode. A plausible explanation is that two groups of bubble are generated under the dual-bubble pumping mode such that it is easier for thermal bubbles to fill up the chamber for effective pumping.

The pumping pressure at zero flow rate with respect to various driving frequencies is measured and plotted in Fig. 10 based on the dual-bubble micropump mode and the flow rate at zero back pressure is shown together for comparison. The maximum measured pressure is $377 \mathrm{~Pa}$ at $5 \%$ pulse duty under an averaged input power of $0.5 \mathrm{~W}$. In experiments, at each driving frequency, the liquid is still oscillating in the polyimide tube and the maximum pressure is recorded when the maximum fluid height at outlet port is achieved. These experimental data have been gathered from the prototype devices. There are two general areas to be further investigated for the optimal design and operation of the bubble-powered, nozzle-diffuser micropump: 1) the geometric optimization of the structure such as the pumping chamber and nozzle-diffuser designs and 2) the bubble generation mechanisms including heat transfer and fluidic analyzes. Olsson et al. [14], [15] have reported detail studies on the modeling and optimization of nozzle-diffuser designs. Although they have used a piezoelectrically-driven, mechanical diaphragm as the actuation source, the analytical and experimental approaches will be extremely useful toward the optimization of the micropump presented in this work. In the area of microscale bubble formation, there have been publications on the static and transient bubble formation characterizations [16], [17] addressing key issues in microscale resistive heating and bubble nucleation. It is believed that the optimization of the thermal-bubble-actuated, nozzle-diffuser micropump can be achieved with further analytical and experimental studies and with the help of these previous works.

\section{APPLICATION IN MiCROFLUIDIC MIXER}

This bubble powered nozzle-diffuser micropump has been used in a microfluidic system to assist mixing of liquids [18]. With proper microchannel design, the oscillatory flows generated by the micropump can be used to induce a wavy interface between two liquids to enlarge the contact surface and accelerate the mixing process.

Fig. 11 illustrates a microfluidic system including a micropump and a mixing channel. This mixing channel is constructed together with the micropump process as shown in Fig. 4 such that no extra manufacturing step is required. Liquids are supplied and pumped from two inlet ports by means of gravity force to supply a flow rate of $6.5 \mu \mathrm{l} / \mathrm{min}$ and the micropump is used to agitate the liquid interface to accelerate the mixing process. Fig. 12 shows the experimental results. The dark color is the blue dye testing liquid coming from liquid inlet port 1 and moving upward in the microchannel that is $200 \mu \mathrm{m}$ in width and of $50 \mu \mathrm{m}$ in depth. The bright color is IPA passing through micropump and coming from liquid inlet port 2. The micropump is connected to the IPA injection channel as shown in Fig. 11. Before the micropump is turned on, there is little mixing effect as shown in Fig. 12(a) where tow liquids are flowing with a speed of about $10 \mathrm{~mm} / \mathrm{sec}$. When the micropump is turned on with a driving frequency of $5 \mathrm{~Hz}$, Fig. 12(b) shows the mixing result when the bubble is expanding. It appears that IPA is pushed out into the blue dye and the well-defined boundary in Fig. 12(a) is broken. During the bubble collapsing process, the boundary of IPA moves back a bit at the intersection of the injection channel and the mixing channel. Hence, a wavy liquid boundary is generated. This effect increases the contact area and assists the mixing effect. Fig. 13 shows a series of pictures, in which the micro pump is driven under various pulse frequencies of, 5, 50, 100, 150, and $200 \mathrm{~Hz}$ from left to right. As it can be seen, the wavelength of the wavy patterns becomes shorter as the driving frequency 


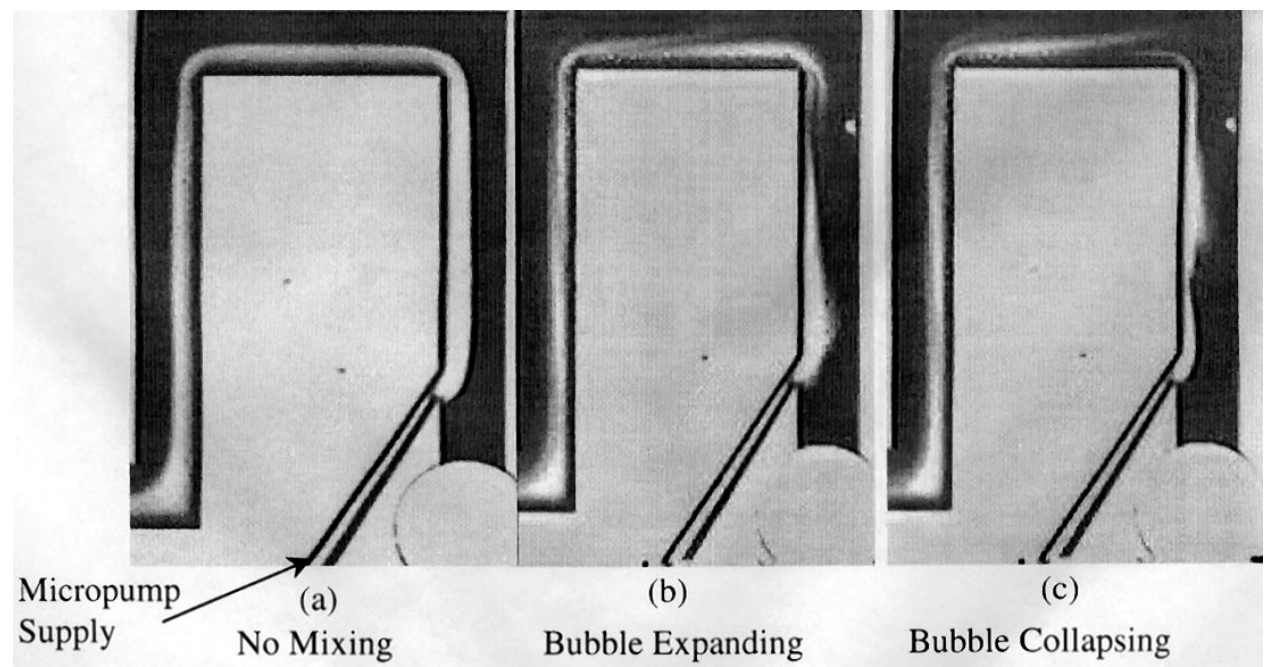

Fig. 12. Wavy liquid interface and interlacing injection effect caused by the micropump agitation at $5 \mathrm{~Hz}$ driven frequency.

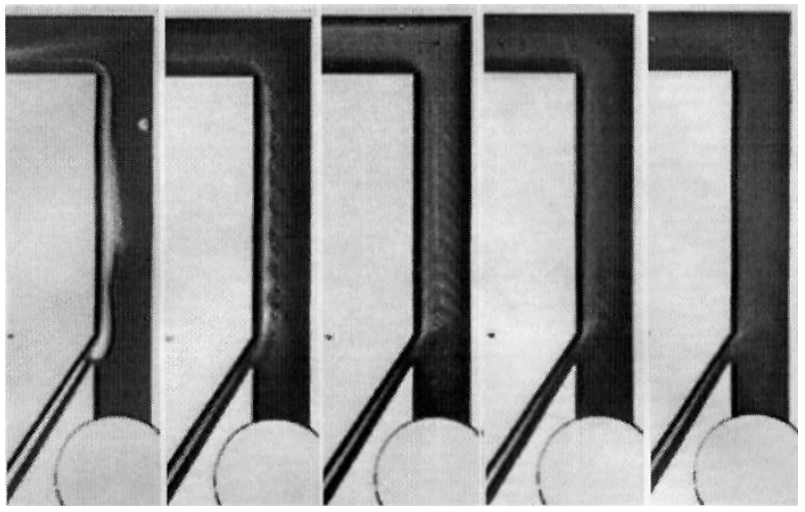

Fig. 13. Mixing of IPA and blue dye near the initial encountering point under various micropump driving frequencies, 5, 50, 100, 150, and $200 \mathrm{~Hz}$ from left to right.

increases and the color of mixing liquids becomes uniform. It indicates that better mixing results are accomplished.

\section{CONCLUSION}

A family of thermal-bubble-actuated micronozzle-diffuser pumps, including single-bubble and dual-bubble pumping modes, are demonstrated. The thermal bubbles generated in the pump are driven by pulsive electrical signals with driving frequencies up to $500 \mathrm{~Hz}$. The measured pumping flow rate can reach $5 \mu \mathrm{l} / \mathrm{min}$ and the highest pumping pressure is 377 Pa. Optimal pulse widths of $0.4 \mathrm{~ms}$ and $0.13 \mathrm{~ms}$ are characterized experimentally for highest pumping rates for single and dual-bubble pumping designs, respectively. It is found that residual bubbles in the pumping chamber reduce the pumping efficiency and pressure head and the design of dual-bubble pumping mode can alleviate the problem. One application is demonstrated in a microfluidic system to use the bubble pump to generate oscillatory flow for microfluidic mixing applications. In summary, this work accomplished the principles of thermal bubble pumping with the help of nozzle-diffuser flow regulation. Further investigations in the areas of microscale heat transfer, phase change, fluid dynamics in the nozzle-diffuser designs and bubble nucleation and collapsing processes are necessary to improve the reliability and performance of thermal-bubble-actuated micronozzle-diffuser pumps.

\section{ACKNOWLEDGMENT}

The authors would thank the Professor Liepmann's research group at the University of California (UC), Berkeley, testing and discussions. These devices are fabricated in the microlab at UC Berkeley.

\section{REFERENCES}

[1] H. Mizoguchi, M. Ando, T. Mizuno, T. Takagi, and N. Nakajima, "Design and fabrication of light driven micropump," in Proc. 1992 IEEE Micro Electro Mechanical Systems Workshop, Travemunde, Germany, 1992, pp. 31-36.

[2] E. Stemme and G. Stemme, "A vavleless diffuser/nozzle-based fluid pump," Sens. Actuators, vol. A39, pp. 159-167, 1993.

[3] B. Bustgens, W. Bacher, W. Menz, and W. K. Schomburg, "Micropump manufactured by thermoplastic molding," in Proc. 1994 IEEE Micro Electro Mechanical Systems Workshop, Oiso, Japan, 1994, pp. 18-21.

[4] T. Jun and C. J. Kim, "Microscale pumping with traversing bubbles in microchannels," in Proc. IEEE Solid-State Sensor Actuator Workshop, Hilton Head Island, SC, 1996, pp. 144-147.

[5] S. F. Bart, L. S. Tavrow, M. Mehregany, and J. H. Lang, "Microfabricated electrohydrodynamic pumps," Sens. Actuators, vol. A21-23, pp. 193-197, 1990.

[6] J. Lee and C. J. Kim, "Liquid micromotor driven by continuous electrowetting," in Proc. 1998 IEEE Micro Electro Mechanical Systems Workshop, Heidelberg, Germany, 1998, pp. 538-543.

[7] N. J. Nielsen, "History of ThInkjet printerhead development," $H P$ Journal, vol. 36, no. 5, pp. 4-10, 1985.

[8] L. Lin and A. P. Pisano, "Thermal bubble powered microactuators," Microsyst. Technol. J., vol. 1, no. 1, pp. 51-58, 1994.

[9] H. T. G. Van Lintel, F. C. M. van de Pol, and S. Bouwstra, "A Piezoelectric micropump based on micromachining of silicon," Sens. Actuators, vol. 15, pp. 153-167, 1988.

[10] M. Esashi, S. Shoji, and A. Nakano, "Normally closed microvalve and micropump fabricated on a silicon wafer," Sens. Actuators, vol. A20, pp. 163-169, 1989.

[11] J. G. Smits, "Piezoelectric micropump with three valves working peristaltically," Sens. Actuators, Phys. A, vol. 21-23, pp. 203-206, 1990.

[12] S. Matsumoto, A. Klein, and R. Maeda, "Development of bi-directional valve-less micropump for liquid," in Proc. 1999 IEEE Micro Electro Mechanical Systems Workshop, Orlando, FL, 1999, pp. 141-146.

[13] F. Forster, R. L. Bardell, M. A. Afromowitz, N. R. Sharma, and A. Blanchard, "Design, fabrication and testing of fixed-valve micro-pumps," in Proc. ASME Fluids Eng. Div., 1995, pp. 39-44.

[14] A. Olsson, "Valve-Less Diffuser Micropumps,", Royal Institute of Technology, Stockholm, Sweden, 1998 
[15] A. Olsson, G. Stemme, and E. Stemme, "A valve-less planar fluid pump with two pump chambers," Sens. Actuators, vol. A46-47, pp. 549-556, 1995.

[16] L. Lin, A. P. Pisano, and V. P. Carey, "Thermal bubble formations on polysilicon micro resistors," ASME J. Heat Transfer, vol. 120, pp. 735-742, Sept. 1998

[17] J. H. Tsai and L. Lin, "Transient thermal bubble formation on a polysilicon micro-resister," ASME J. Heat Transfer, vol. 124, no. 2, pp. 375-382, 2002

[18] _ - "Active microfluidic mixer and gas bubble filter driven by thermal bubble micropump," Sens. Actuators, vol. A97-98, pp. 665-671, 2002.

Jr-Hung Tsai received the M.S. degree in power mechanical engineering from the National Tsing Hua University at Taiwan in 1996 and the Ph.D. degree in mechanical engineering from the University of Michigan, Ann Arbor, in 2001.

He is currently with Kumetrix, Inc., CA, as a a research staff member. His major research interests are in design, modeling, and fabrication of microfluidic components, microbiosensors, and microactuators in microfluidic systems.

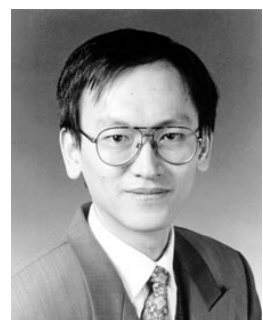

Liwei Lin (S'92-M'93) received the M.S. and $\mathrm{Ph} . \mathrm{D}$. degrees in mechanical engineering from the University of California, Berkeley, in 1991 and 1993, respectively.

From 1993 to 1994, he was with BEI Electronics, Inc., in research and development of microsensors. From 1994 to 1996, he was an Associate Professor in the Institute of Applied Mechanics, National Taiwan University, Taiwan. From 1996 to 1999, he was an Assistant Professor at the Mechanical Engineering and Applied Mechanics Department at the University of Michigan. In 1999, he joined the University of California at Berkeley and is now an Associate Professor at Mechanical Engineering Department and Co-Director at Berkeley Sensor and Actuator Center, NSF/Industry/University research cooperative center. His research interests are in design, modeling and fabrication of microstructures, microsensors, and microactuators as well as mechanical issues in microelectromechanical systems including heat transfer, solid/fluid mechanics, and dynamics. He holds seven U.S. patents in the area of MEMS.

Dr. Lin is the recipient of the 1998 NSF CAREER Award for research in MEMS Packaging and the 1999 ASME Journal of Heat Transfer best paper award for his work on microscale bubble formation. He led the effort in establishing the MEMS subdivision in ASME and is currently serving as the Vice Chairman of the Executive Committee for the MEMS subdivision. 